\title{
Pressure and temperature-based adaptive observer of air charge for turbocharged diesel engines
}

\author{
A. G. Stefanopoulou ${ }^{1, *, \dagger}$, O. F. Storset ${ }^{2}$ and R. Smith $^{2}$ \\ ${ }^{1}$ University of Michigan, Ann Arbor,U.S.A. \\ ${ }^{2}$ University of California, Santa Barbara,U.S.A.
}

\begin{abstract}
SUMMARY
In this paper we design an adaptive air charge estimator for turbocharged diesel engines using intake manifold pressure, temperature and engine speed measurements. This adaptive observer scheme does not depend on mass air flow sensors and can be applied to diesel engines with no exhaust gas recirculation (EGR). The performance of the adaptive scheme is shown in simulations to be comparable to conventional air charge estimation schemes if perfect temperature measurements are available. The designed scheme cannot estimate fast transients and its performance deteriorates with temperature sensor lags. Despite all these difficulties, this paper demonstrates that (i) the proposed scheme has better robustness to modelling errors because it provides a closed-loop observer design, and (ii) robust air charge estimation is achievable even without air flow sensors if good (fast) temperature sensors become available. Finally, we provide a rigorous proof and present the implementation challenges as well as the limiting factors of this adaptation scheme and point to hardware and temperature sensor requirements. Copyright (C) 2004 John Wiley \& Sons, Ltd.
\end{abstract}

KEY WORDS: estimation; adaptive; nonlinear; engine; sensors; automotive; emissions

\section{INTRODUCTION}

Air charge estimation is an integral part of modern automotive controllers and a critical algorithm for low emission vehicles. In gasoline throttled port fuel injection engines air charge estimation is used to schedule the fuel injection command that will result in a cylinder mixture with stoichiometric air-to-fuel ratio. The air-to-fuel ratio regulation to the stoichiometric fuel value is a stringent requirement that ensures high conversion of the $\mathrm{CO}, \mathrm{HC}$, and $\mathrm{NO}_{x}$ feedgas pollutants to less harmful tailpipe emissions through the three-way-catalytic (TWC) converter [1]. The fuel scheduling is typically controlled via a combination of a measured AFR (feedback)

\footnotetext{
*Correspondence to: A. G. Stefanopoulou, Mechanical Engineering, University of Michigan, G058 WE Lay Auto Lab, 1231 Beal Ave, Ann Arbor, MI 48109-2121,U.S.A.

†E-mail: annastef@umich.edu

Contract/grant sponsor: National Science Foundation; contract/grant number: NSF-ECS-0049025

Contract/grant sponsor: National Science Foundation; contract/grant number: NSF-ECS-9978562
}

Published online 10 February 2004

Copyright (C) 2004 John Wiley \& Sons, Ltd. 
signal and an estimated cylinder air charge (feedforward) signal. Seminal contributions can be reviewed in References [2-5] and the references therein.

Air charge estimation is used in diesel engines to limit the fuel flow command and avoid low AFR. Low AFR is undesirable because it results in visible smoke and excessive particulate matter. Turbocharged diesel engines typically operate with very lean mixtures $(A F R>35)$ thus the fuel is scheduled to meet the driver torque demand and road load. During fast accelerations or sudden load changes the scheduled fuel flow can cause rich AFR excursions $\left(\mathrm{AFR}<\mathrm{AFR} \mathrm{sl}_{\mathrm{sl}}=\right.$ 25) and consequently visible smoke generation. The rich AFR excursions last until the air flow adjusts to a new higher value due to the increase of the exhaust gas energy, and consequent increase in turbocharger speed and compressor flow [6]. This transient excursion can be avoided if an accurate air charge estimator is used to trigger an upper limit to the fuel flow that keeps the actual AFR above the smoke limit [7].

The difficulty is mostly during transients and requires characterisation of the engine breathing dynamics [8] to analyse and develop real-time algorithms [9]. Most of the estimation and control algorithms are similar to the ones developed for throttled gasoline engines taking only into account the differences due to the turbocharger and intercooler dynamics. The air charge estimation is based again on a static volumetric efficiency map that depends on intake manifold (boost) pressure and engine speed. During transient fueling changes however, there are very large deviations of the instantaneous ratio between the exhaust and intake manifold pressure from their steady-state values. These transient deviations have significant effects to the value of the engine breathing capacity and its air charge estimation. Characterisation of this dynamic breathing behaviour requires the additional parameterisation of volumetric efficiency with respect to exhaust manifold pressure $[10,11]$.

Hence additional sensors are considered. In Reference [12] an exhaust manifold pressure sensor is introduced in addition to an intake manifold pressure sensor to facilitate the prediction of the transient breathing characteristics. The complexity introduced by the varying intake manifold temperature is addressed in Reference [13] and the authors indicate the benefits of fast temperature measurement. Additional measurements such as a universal (linear) exhaust gas oxygen (EGO) sensor for AFR feedback in Reference [14] and closed-loop air charge estimation in Reference [15] are also investigated.

Another important collection of work on air charge estimation is proposing the use of adaptive observers for on-line estimation of the engine breathing characteristics. This approach is especially desirable due to the reduced engine mapping requirements and thus easy vehicle calibration and low development cost [16]. It is also necessary during significant aging and parameter variations [15]. Moreover, it has been proposed in order to account for the engine dynamic behaviour during fast transients after appropriate parameterisation [17-19]. All the above efforts depend on traditional sensing scheme of speed, intake manifold pressure, and inlet air flow.

In this paper an adaptive air charge estimation scheme is presented that uses intake manifold temperature sensors instead of the expensive and delicate air flow sensor. The proposed scheme can only work for engines with no EGR. Indeed, EGR changes the intake mass composition and requires additional measurements. After a few preliminary notes for the engine model dynamics, the measurements and the system observability are presented in Section 2, the algorithm is presented in Section 3. The proof delineates the difficulties arising from the slow temperature sensors and unmodelled sensor dynamics in Section 3.1. A simulation of the estimation scheme assuming a fast temperature sensor (with 100-200 ms time constant) is 
included in Section 4 to allow comparison with the traditional estimation schemes. Although such fast thermocouples are not available currently, we believe that it is important to show what is achievable with this scheme if fast temperature measurements become available in the future.

\section{PRELIMINARIES}

In the sequel, $(\cdot)$ denotes a measured variable, or a variable constructed from measurements only, so that $\bar{x}$ is the measurement of $x$. The notation $(\hat{*})$ is used for estimated variables and the $(\because)$ is used for the error in the estimated variables. The set of positive real numbers excluding zero is denoted by $\mathbb{R}_{+}, \dot{x}$ denotes $(\mathrm{d} / \mathrm{d} t) x$, and $\mathscr{P}^{n}(x)$ is an $n$th order polynomial in $x$. The operator $[H u](t, N)$ denotes the filter with the output $y=C(N(t)) x+D(N(t)) u$ with $\dot{x}=A(N(t)) x+B \times$ $(N(t)) u$. For convenience, the dependency on the time-varying signal $N(t)$ will be omitted so that $[H u](t)=[H u](t, N)$. Similarly, we will omit the time dependence on signals so that $u=u(t)$, $N=N(t)$, etc. A complete list of all the variables used is in Appendix B. Some of the physical variables are shown in Figure 1 and we define them in the following text when they are first introduced.

\subsection{Model and estimation schemes}

The engine is approximated as a 'continuous flow device' such as a pump. The objective of this paper is to develop an accurate estimate for the air flow through the engine $W_{1 \mathrm{e}}$ that is assumed to be constant during a cycle and is given by

$$
W_{\mathrm{le}}=k_{1}^{2} k_{3} N m_{1} \eta_{\mathrm{v}}, \quad k_{3}=\frac{V_{\mathrm{d}}}{R_{1} 120}, \quad k_{1}=\sqrt{\frac{R_{1}}{V_{1}}}
$$

where $R_{1}$ is the air gas constant, $V_{1}$ is the volume of the intake manifold, $V_{\mathrm{d}}$ is the engine total displacement volume, $m_{1}$ is the mass in the intake manifold and $\eta_{\mathrm{v}}$ is the volumetric efficiency $[10,11]$ :

$$
\eta_{\mathrm{v}}=\eta_{\rho}\left(\frac{p_{2}}{p_{1}}\right) \eta_{z}\left(N, \sqrt{T_{1}}\right)
$$

where $\eta_{\rho}$ accounts for the pumping losses due to different pressures in the exhaust $\left(p_{2}\right)$ and intake $\left(p_{1}\right)$ manifold. The term $\eta_{z}$ accounts for the effects of the piston speed which depends on

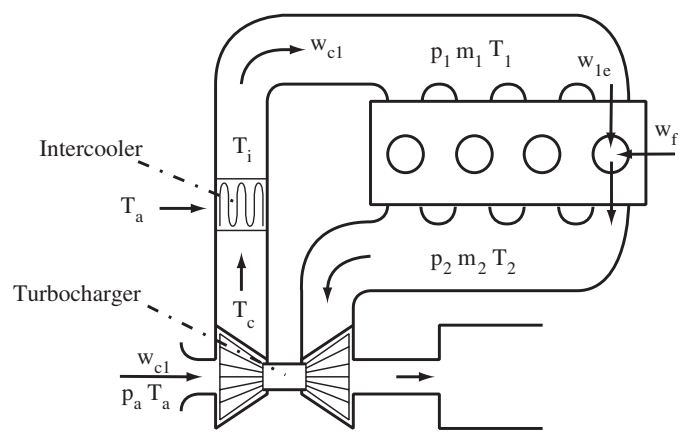

Figure 1. A turbocharged diesel engine with intercooler. 
the engine speed $(N)$ and the velocity of sound in the intake manifold (Mach number) through the temperature $\left(T_{1}\right)$.

This model is clearly not valid for short time intervals because it disregards the cylinder-tocylinder events, but it has good accuracy on time scales slightly larger than an engine cycle $[8,20,21]$. The energy balance (Equation (3)) and mass balance (Equation (4)) in the intake manifold given adiabatic conditions result in the state equation for pressure, $p_{1}$, and mass, $m_{1}$, respectively. They are related with the intake manifold temperature, $T_{1}$, through the ideal gas law (5):

$$
\begin{gathered}
\dot{p}_{1}=\kappa k_{1}^{2}\left(W_{\mathrm{cl}} T_{\mathrm{i}}-W_{\mathrm{le}} T_{1}\right) \\
\dot{m}_{1}=W_{\mathrm{cl}}-W_{\mathrm{le}} \\
T_{1}=\frac{1}{k_{1}^{2}} \frac{p_{1}}{m_{1}}
\end{gathered}
$$

where $\kappa$ is the ratio of specific heats and $T_{\mathrm{i}}$ the intercooler temperature that depends on compressor and intercooler efficiencies and is different from $T_{1}$ during transients. The model described by Equations (1)-(5) is used after several simplifications and assumptions in all the existing implementations of air charge estimation. These schemes and their related measurements are summarised below and serve as basis for comparison.

Modelling the air charge and the cylinder air flow is simplified in throttled engines because the intake manifold air temperature and the air inlet temperature are considered constant and equal to the ambient temperature $\left(T_{1}=T_{\mathrm{i}}=T_{\mathrm{a}}\right)$. This eliminates one of the two state equations (3)(4) and simplifies the intake manifold filling dynamics. Moreover, the volumetric efficiency can be simplified and represented as a function of the intake manifold pressure and engine speed, only: $\eta_{\mathrm{v}}=\mathscr{P}_{\mathrm{v}}\left(N, p_{1}\right)$. This function can be derived using engine flow measurements during steady-state conditions.

In the 'MAP'-based air charge estimation scheme the key measurement is the intake manifold absolute pressure (MAP) that provides exact pressure measurements, i.e. $\bar{p}_{1}=p_{1}$. Temperature is measured with conventional (slow) sensors providing $\bar{T}_{1}$. It is almost always true that $\bar{N}=N$, and thus, $\hat{\eta}_{\mathrm{v}}=\mathscr{P}_{\mathrm{v}}\left(N, \bar{p}_{1}\right)$. Open-loop estimation is used to calculate the engine air flow using (1) and (5):

$$
\hat{W}_{1 \mathrm{e}}=k_{3} N \frac{\bar{p}_{1}}{\bar{T}_{1}} \mathscr{P}_{\mathrm{v}}\left(N, \bar{p}_{1}\right)
$$

In addition to modelling errors in $\mathscr{P}_{\mathrm{v}}$ that affects both transient and steady-state estimated air charge, this scheme is prone to temperature variations.

In the 'MAF'-based estimation scheme we assume perfect measurement of the mass air flow (MAF) into the manifold $\bar{W}_{\mathrm{cl}}=W_{\mathrm{cl}}$ (or $\bar{W}_{\mathrm{thr}}=W_{\mathrm{thr}}$ for throttled engines). This scheme either assumes (i) that the difference between $W_{\mathrm{le}}$ and $W_{\mathrm{cl}}$ is negligible and thus uses $W_{\mathrm{le}}=\bar{W}_{\mathrm{cl}}$ even during transients, or (ii) utilises dynamic compensation for the manifold filling dynamics based on a map of the steady-state air flow $\mathscr{P}_{w}\left(N, p_{1}, T_{1}\right)$ as shown in Reference [22]:

$$
\begin{gathered}
\dot{\hat{p}}_{1}=\kappa k_{1}^{2} T_{1}\left(\bar{W}_{\mathrm{cl}}-\mathscr{P}_{w}\left(N, \hat{p}_{1}, \bar{T}_{1}\right)\right) \\
\hat{W}_{\mathrm{le}}=\mathscr{P}_{w}\left(N, \hat{p}_{1}, \bar{T}_{1}\right)\left(=k_{3} N \frac{\hat{p}_{1}}{\bar{T}_{1}} \mathscr{P}_{\mathrm{v}}\left(N, \hat{p}_{1}\right)\right)
\end{gathered}
$$


Errors in $\mathscr{P}_{w}\left(N, p_{1}, T_{1}\right)$ and the assumption of constant (or slowly varying) $T_{1}$ will cause errors during transients.

The two major simplifications that the traditional air charge estimation schemes make are:

(a) Volumetric efficiency and consequently engine air flow do not depend on exhaust manifold pressure (downstream pressure).

(b) Intake manifold temperature is constant.

Volumetric efficiency (item (a)) in turbocharged diesel engines is a function of $p_{2}$. To circumvent the $p_{2}$ based parameterisation in Equation (2), we will assume that the volumetric efficiency depends on $N$ and a time-varying parameter $\theta(t)$ that needs to be identified so that

$$
\eta_{\mathrm{v}}(t)=\mathscr{P}_{\rho}(N(t)) \theta(t)
$$

where $\mathscr{P}_{\rho}(N)>0$ is a polynomial in $N$ that accounts for the pumping rate's dependency on engine speed. The variable $\theta(t)$ is an unknown time-varying coefficient that accounts for all the other phenomena mentioned. Note that in TC diesel engines we have to assume that $\theta(t)$ is a time-varying parameter because of the fast variations of $p_{2} / p_{1}$. In throttled engines we can assume that $\theta(t)$ is an unknown constant or a slow-varying parameter as in References $[15,16]$.

The fact that mass and pressure are independent during transients is usually neglected in conventional estimation schemes where $T_{\mathrm{i}}$ and $T_{1}$ are assumed equal and constant (item (b)). This variability is represented in Equations (3)-(4) and the model we use after the volumetric efficiency parameterisation becomes

$$
\begin{gathered}
\dot{p}_{1}=-\kappa k_{1}^{2} k_{3} N \mathscr{P}_{\rho}(N) \theta p_{1}+\kappa k_{1}^{2} W_{\mathrm{c} 1} T_{\mathrm{i}} \\
\dot{m}_{1}=-k_{1}^{2} k_{3} N \mathscr{P}_{\rho}(N) \theta m_{1}+W_{\mathrm{c} 1}
\end{gathered}
$$

We use pressure and temperature measurements $\left(T_{\mathrm{i}}\right.$ and $\left.T_{1}\right)$ for on-line estimation of $\theta(t)$ and then create a closed-loop observer for $m_{1}$ that is needed for the air flow estimation (Equation (1)). The observability of the system states with this sensing scheme is presented in Section 2.3.

\subsection{Measurements}

Sensor selection in engines depends on their cost, reliability and precision. The engine speed $N$ is assumed measured throughout the sequel, and it is precise so we assume $\bar{N}=N$. The flow into the intake manifold, $W_{\mathrm{cl}}$, is typically measured with a hot wire anemometer, $\bar{W}_{\mathrm{cl}}$, but its performance deteriorates with use even for expensive devices. This is why we explore its replacement if fast temperature sensors become available. Intake manifold pressure, $p_{1}$, can be measured precisely with a large bandwidth at moderate cost relative to $W_{\mathrm{cl}}$. However, the engine events cause pressure fluctuations so that $\bar{p}_{1}=p_{1}+\Delta p_{1}$, where $\Delta p_{1}$ represents the cylinder-to-cylinder flow events and the unmodelled dynamics which are not present in the mean value model. The term $\Delta p_{1}$ typically has a rectified sinusoidal shape with frequency of the sinusoid equal to $2 \pi N / 60 \mathrm{rad} / \mathrm{s}$ appearing as measurement 'noise' which might destabilize the identified scheme for the parameter $\theta(t)$.

Temperature measurements are typically conducted with thermocouples which have a time constant varying with the flow of air. The fastest thermocouples are significantly slower than $p_{1}$ measurements, so temperature measurements limit the observer bandwidth. However, there is significant development in automotive sensors from the progression of microelectro-mechanical 
systems (MEMS) which might result in higher bandwidth automotive temperature sensors in the future. Note here that in experimental configurations, one can use co-axial thermocouples [23] to obtain fast response metal wall surface temperature measurements.

The temperature of air leaving the intercooler, $T_{\mathrm{i}}$, can be assumed to be slowly time varying due to the high efficiency of air-to-air intercoolers and the slowly varying ambient temperature [24]. For better precision a measurement has to be taken. The cost versus precision considerations are the same as for the $T_{1}$ measurement.

\subsection{Observability based on temperature measurements}

The issue of observability for a stable plant addresses whether it is possible to create an observer whose state estimate converges faster to the actual state than the plants dynamics, given perfect knowledge of the plant, the inputs and the outputs except its present state. In the linear case the famous Kalman rank condition can be used to check if the plant is observable. However, for a general nonlinear system there is no generic way of checking observability, and the observer structure is unknown. For completeness we review here some definitions of nonlinear observability [25].

Observability for the system $x=f(x, u)$ with $y=h(x, u)$ with state $x \in \mathscr{D}$, control input $u \in \mathscr{U}$ and solution $\Phi\left(x\left(t_{0}\right), u, t\right)$ at time $t$ from the initial condition $x\left(t_{0}\right)$, can be defined with the concept of indistinguishable initial conditions.

\section{Definition 1 (Indistinguishable states)}

The initial condition pair $\left(x\left(t_{0}\right), x^{\prime}\left(t_{0}\right)\right), x\left(t_{0}\right) \neq x^{\prime}\left(t_{0}\right)$, is said to be indistinguishable by $u$ if $h\left(\Phi\left(x\left(t_{0}\right), u, t\right), u\right)=h\left(\Phi\left(x^{\prime}\left(t_{0}\right), u, t\right), u\right) \forall t \geqslant t_{0}$. If the pair is indistinguishable by all $u$; it is said to be indistinguishable.

\section{Definition 2 (Observability)}

A nonlinear system is observable if it does not have any indistinguishable pairs of states.

Note that observability of a nonlinear system does not exclude the possibility of states indistinguishable by some inputs. So observability is in general not enough to be able to design a closed-loop observer. There have to be additional constraints on the input. Such an input is called a universal input:

\section{Definition 3 (Universal input)}

An input $u \in \mathscr{U}$ is universal on $\left[t_{0}, t\right]$ if for every pair of distinct states $x\left(t_{0}\right)$ and $x^{\prime}\left(t_{0}\right)$ there exists $\tau \in\left[t_{0}, t\right]$ such that $h\left(\Phi\left(x\left(t_{0}\right), u, \tau\right), u\right) \neq h\left(\Phi\left(x^{\prime}\left(t_{0}\right), u, \tau\right), u\right)$. If $u$ is universal for all $t>0$ it is just said to be universal.

A non-universal input is called a singular input. If the system is observable and U only contains universal inputs, it is possible to create an observer whose state converges faster to the actual state than the plants dynamics. Such a plant is said to be uniformly observable.

Definition 4 (Uniformly observable (UO))

A system that is observable and all inputs $u$ are universal is said to be uniformly observable. 
It should be emphasised that even if a system is UO, the observer structure is unknown for a general nonlinear system. In order to evaluate the observability properties of a system directly from the definition of indistinguishable states, it is necessary to have the explicit solution $\Phi\left(x\left(t_{0}\right), u, t\right)$ which is rarely available for nonlinear systems. It is therefore in general not possible to check observability directly by using the definition of indistinguishable states.

In our case to estimate $\hat{W}_{1 \mathrm{e}}$ we need to produce an estimate of $m_{1}$ since it cannot be measured directly. Observability of $m_{1}$ can then be assessed from $p_{1}$ and/or $T_{1}$ measurements assuming correct system equations, (10)-(11), and perfect knowledge of all the model parameters $\eta_{\mathrm{v}}$ and $W_{\mathrm{cl}}, T_{\mathrm{i}}, N$ as follows. Equations (10)-(11) and (5) can be written

$$
\begin{gathered}
{\left[\begin{array}{c}
\dot{p}_{1} \\
\dot{m}_{1}
\end{array}\right]=\left[\begin{array}{c}
-\kappa k_{1}^{2} k_{3} N(t) \eta_{\mathrm{v}}(t) p_{1}+\kappa k_{1}^{2} W_{\mathrm{cl}} T_{\mathrm{i}} \\
-k_{1}^{2} k_{3} N(t) \eta_{\mathrm{v}}(t) m_{1}+W_{\mathrm{c} 1}
\end{array}\right]} \\
T_{1}=\frac{1}{k_{1}^{2}} \frac{p_{1}}{m_{1}}
\end{gathered}
$$

where $x^{\mathrm{T}}=\left[p_{1}, m_{1}\right]$ is the state and $T_{1}$ is the output. Equation (12) is a nonlinear system. However, if we consider $u^{\prime \mathrm{T}}=\left[W_{\mathrm{cl}} T_{\mathrm{i}}, W_{\mathrm{cl}}\right]$ any input and $N(t)$ as the known model parameter, the state equation (12) can be viewed as a linear time-varying system

$$
\begin{gathered}
{\left[\begin{array}{c}
\dot{p}_{1} \\
\dot{m}_{1}
\end{array}\right]=\left[\begin{array}{cc}
-\kappa a(t) & 0 \\
0 & -a(t)
\end{array}\right]\left[\begin{array}{l}
p_{1} \\
m_{1}
\end{array}\right]+\left[\begin{array}{cc}
\kappa k_{1}^{2} & 0 \\
0 & 1
\end{array}\right]\left[\begin{array}{c}
W_{\mathrm{c} 1} T_{\mathrm{i}} \\
W_{\mathrm{c} 1}
\end{array}\right]} \\
a(t)=k_{1}^{2} k_{3} N(t) \eta_{\mathrm{v}}(t)
\end{gathered}
$$

with a nonlinear output equation if $y=h(x)=T_{1}$ given by (13).

If we denote $\Phi\left(x\left(t_{0}\right), u, t\right)$ the solution to (14) at time $t$ with initial condition $x\left(t_{0}\right)$ with input $u$, the output becomes

$$
\begin{aligned}
T_{1}(t) & =g_{1}\left(\Phi\left(x\left(t_{0}\right), u, t\right)\right) \\
& =: \frac{1\left(\Phi_{1}\left(t, t_{0}\right)\right)^{\kappa} p_{1}\left(t_{0}\right)+\kappa k_{1}^{2} \int_{t_{0}}^{t}\left(\Phi_{1}(t, \tau)\right)^{\kappa} W_{\mathrm{c} 1} T_{\mathrm{i}}(\tau) \mathrm{d} \tau}{k_{1}^{2} \quad \Phi_{1}\left(t, t_{0}\right) m_{1}\left(t_{0}\right)+\int_{t_{0}}^{t} \Phi_{1}(t, \tau) W_{\mathrm{cl}} \mathrm{d} \tau}
\end{aligned}
$$

where the state transition matrix is

$$
\Phi(t, \tau)=\mathrm{e}^{-k_{1}^{2} k_{3} \int_{\tau}^{t} N(\sigma) \eta_{\mathrm{v}}(\sigma) \mathrm{d} \sigma}
$$

Thus, system (2) is uniformly observable (UO) from (13) because there no states $x\left(t_{0}\right) \neq x^{\prime}\left(t_{0}\right)$ such that $g_{1}\left(\Phi\left(x\left(t_{0}\right), u, t\right)\right)=g_{1}\left(\Phi\left(x^{\prime}\left(t_{0}\right), u, t\right)\right)$ for all time and all possible inputs. Note that if we consider $y=p_{1}$ the output equation (13) does not contain $m_{1}$, and system (12) is decoupled, thus observability is lost.

If both $p_{1}$ and $T_{1}$ are measured, system (12)-(13) is obviously observable since it is so in the case when only $T_{1}$ is measured. However, it is beneficial to consider this case to see the effects of the additional measurement. In particular, it is interesting to see that the two measurements together result in a linear time-varying system. We can now disregard the $\dot{p}_{1}$ equation in the observability assessment since we measure $p_{1}$, and there is no coupling between the states. 
$T_{1}$ must now be considered an input, and the system becomes

$$
\begin{gathered}
\dot{m}_{1}=-a(t) m_{1}+W_{\mathrm{cl}} \\
p_{1}=k_{1}^{2} T_{1} m_{1}=: C(u) m_{1}
\end{gathered}
$$

which is a linear time-varying system. The state transition matrix for this system is (16), and the observability grammian becomes

$$
\begin{aligned}
\mathcal{O}(t, T, u) & =\int_{t}^{t+T} \Phi^{\mathrm{T}}(\tau, t) C^{\mathrm{T}}(u(\tau)) C(u(\tau)) \Phi(\tau, t) \mathrm{d} \tau \\
& =k_{1}^{4} \int_{t}^{t+T} T_{1}^{2}(\tau) \mathrm{e}^{-2 k_{1}^{2} k_{3} \int_{t}^{\tau} N(\sigma) \eta_{\mathrm{v}}(\sigma) \mathrm{d} \sigma} \mathrm{d} \tau
\end{aligned}
$$

Since $T_{1}, N$ and $\eta_{\mathrm{v}}$ are positive $\forall t$ we have that $\mathcal{O}(t, T, u)>0 \forall T>0$, and $\forall u=\left[W_{\mathrm{cl}}, T_{\mathrm{i}}, N, T_{1}\right]$ in the corresponding input space, and we can conclude that $m_{1}$ is $\mathrm{UO}$ from $y^{\mathrm{T}}=\left[p_{1}, T_{1}\right]$.

Note here that although $p_{1}$ is measured and thus we can disregard the $\dot{p}_{1}$ equation in the observability assessment, the actual measurement $\bar{p}_{1}$ has additional fluctuations that can destabilize the adaptive scheme. Owing to these fluctuations we use a feedback observer for $p_{1}$ and do not disregard the $p_{1}$ dynamics from the adaptive observer.

\section{ADAPTIVE OBSERVER SCHEME}

Since $W_{\mathrm{c} 1}$ is an expensive and often imprecise measurement, it is desirable to develop a scheme that does not rely on it. Consequently, neither the identification scheme nor the observer can utilize this signal. This is possible since a parameterisation of the plant independent of $W_{\mathrm{c} 1}$ can be derived in any of the co-ordinates $\left(p_{1}, m_{1}\right),\left(p_{1}, T_{1}\right)$ or $\left(m_{1}, T_{1}\right)$. By constructing the measurement $\bar{m}_{1}=\left(1 / k_{1}^{2}\right) \frac{\bar{p}_{1}}{\bar{T}_{1}}$ from the ideal gas law (5), it is possible to realize the equations for the identifier and the observer. For compactness of presentation, this scheme will only be presented in the co-ordinates $x^{\mathrm{T}}:=\left[p_{1}, m_{1}\right]$, but by using the same methodology it is possible to derive similar schemes in the two other co-ordinates mentioned.

In the observer, $W_{\mathrm{cl}}$ is replaced with a constant $W$, and the resulting error is cancelled with feedback from the measurements. The addition of the $T_{1}$ measurement is essential since the states are not observable from $p_{1}$ alone. The benefits of the extra measurement are a closed-loop observer whose error dynamics converge faster and are less sensitive to modelling errors than an open-loop observer. This identification scheme is sensitive to errors in $\bar{T}_{\mathrm{i}}$ since the cancellation of $W_{\mathrm{cl}}$ in the parameterisation makes $\bar{T}_{\mathrm{i}}$ a factor in the identification error. Consequently, $T_{\mathrm{i}}$ must be measured, so the total measurements become: $p_{1}, T_{1}, T_{\mathrm{i}}$ and $N$.

Using the parameterisation $\eta_{\mathrm{v}}=\mathscr{P}_{z}(N) \theta$ and adding estimation error injection to Equations (10) and (11) and replacing all signals with their measurements $\left(\bar{p}_{1}, \bar{T}_{\mathrm{i}}, \bar{m}_{1}=\left(1 / k_{1}^{2}\right) \bar{p}_{1} / \bar{T}_{1}\right)$, and $\bar{W}_{\mathrm{cl}}=$ const. $=W$ (any value would work) the observer becomes

$$
\begin{gathered}
\dot{\hat{p}}_{1}=-\kappa k_{1}^{2} k_{3} N \mathscr{P}_{z}(N) \hat{\theta} \bar{p}_{1}+\kappa k_{1}^{2} W \bar{T}_{\mathrm{i}}+g_{p 1}\left(\bar{p}_{1}-\hat{p}_{1}\right) \\
\dot{\hat{m}}_{1}=-k_{1}^{2} k_{3} N \mathscr{P}_{z}(N) \hat{\theta} \bar{m}_{1}+W+g_{m 1}\left(\bar{m}_{1}-\hat{m}_{1}\right) \\
\hat{W}_{1 \mathrm{e}}=k_{1}^{2} k_{3} N \mathscr{P}_{z}(N) \hat{\theta} \hat{m}_{1}
\end{gathered}
$$

where, $g_{p 1}$ and $g_{m 1}$ are observer gains to be determined in the design process. 
After we multiply (11) with $\kappa k_{1}^{2} T_{\mathrm{i}}$ and subtract (10) with the parameterisation $\eta_{\mathrm{v}}=\mathscr{P}_{z}(N) \theta$ we derive a reliable identification scheme that is linear in $\theta$ and can guarantee convergence of $\tilde{\theta}$ to 0 :

$$
\begin{gathered}
\kappa k_{1}^{2} T_{\mathrm{i}} \dot{m}_{1}-\dot{p}_{1}=\kappa k_{1}^{2} k_{3} N \mathscr{P}_{z}(N) p_{1} \theta-\kappa k_{1}^{4} k_{3} N \mathscr{P}_{z}(N) m_{1} T_{\mathrm{i}} \theta \\
=-\kappa k_{1}^{2} k_{3} N \mathscr{P}_{z}(N)\left(k_{1}^{2} m_{1} T_{\mathrm{i}}-p_{1}\right) \theta \\
=:-G_{p N}\left(k_{1}^{2} m_{1} T_{\mathrm{i}}-p_{1}\right) \theta
\end{gathered}
$$

A filter $H_{\mathrm{f}}$ with cutoff frequency linear in $N(t)$ defined as

$$
\left[H_{\mathrm{f}} u\right](t)=x_{\mathrm{f}} \quad \text { with } \dot{x}_{\mathrm{f}}=-k_{\omega} N x_{\mathrm{f}}-k_{\omega} N u
$$

with $k_{\omega}>0$ constant, is used to avoid the pure derivatives in (22). Next, define the signal $[\phi \theta](t)$ :

$$
\begin{gathered}
{\left[H_{\mathrm{f}}\left(\kappa k_{1}^{2} T_{\mathrm{i}} \dot{m}_{1}-\dot{p}_{1}\right)\right]} \\
=-\left[H_{\mathrm{f}} G_{p N}\left(k_{1}^{2} m_{1} T_{\mathrm{i}}-p_{1}\right) \theta\right]=:[\phi \theta]
\end{gathered}
$$

and its implementable versions $z(t)$ and $\hat{z}(t)$ :

$$
\begin{aligned}
& z(t):=[\bar{\phi} \theta](t)=-\left[H_{\mathrm{f}} G_{p N}\left(k_{1}^{2} \bar{m}_{1} \bar{T}_{\mathrm{i}}-\bar{p}_{1}\right) \theta\right](t) \\
& \hat{z}(t):=[\bar{\phi} \hat{\theta}](t)=-\left[H_{\mathrm{f}} G_{p N}\left(k_{1}^{2} \bar{m}_{1} \bar{T}_{\mathrm{i}}-\bar{p}_{1}\right) \hat{\theta}\right](t)
\end{aligned}
$$

that are used for the identification error $\varepsilon(t)$ :

$$
\begin{aligned}
\varepsilon(t):=z(t)-\hat{z}(t) & =-\left[H_{\mathrm{f}} G_{p N}\left(k_{1}^{2} \bar{m}_{1} \bar{T}_{\mathrm{i}}-\bar{p}_{1}\right) \tilde{\theta}\right](t) \\
& =\bar{\phi}(t) \tilde{\theta}(t)-\left[H_{\mathrm{s}} \bar{\phi}(\tau) \dot{\tilde{\theta}}(\tau)\right](t) \\
& =\bar{\phi}(t) \tilde{\theta}(t)-\epsilon_{\mathrm{s}}
\end{aligned}
$$

with the regressor

$$
\bar{\phi}(t)=-\left[H_{\mathrm{f}} G_{p N}\left(k_{1}^{2} \bar{m}_{1} \bar{T}_{\mathrm{i}}-\bar{p}_{1}\right)\right](t)
$$

which is linear in the parameter error $\tilde{\theta}$ if we ignore the swapping error $\varepsilon_{\mathrm{s}}=\left[H_{\mathrm{s}} \bar{\phi}(\tau) \dot{\tilde{\theta}}(\tau)\right]$ that arises from pulling $\tilde{\theta}$ out of the filter $H_{\mathrm{f}}$ in (30) using Morse's swapping lemma [26]. The filter $H_{\mathrm{s}}$ in the swapping lemma is defined by

$$
\left[H_{\mathrm{s}} u\right](t):=x_{\mathrm{s}} \quad \text { with } \dot{x}_{\mathrm{s}}=-k_{\omega} N x_{\mathrm{s}}+u
$$

In Appendix A it is shown that we can obtain an implementable identification error $\bar{\varepsilon}$ :

$$
\bar{\varepsilon}(t)=\kappa k_{1}^{2}\left[H_{\mathrm{f}} \bar{T}_{\mathrm{i}}\left(\frac{\mathrm{d}}{\mathrm{d} \tau}+g_{m 1}\right)\left(\bar{m}_{1}-\hat{m}_{1}\right)\right]\left[H_{\mathrm{f}}\left(\frac{\mathrm{d}}{\mathrm{d} \tau}+g_{p 1}\right)\left(\bar{p}_{1}-\hat{p}_{1}\right)\right]
$$

based on measured and estimated variables. The identification error $\bar{\varepsilon}$ is linear in the parameter error $\tilde{\theta}$ except from some terms that depend on the pressure ripples $\Delta p_{1}=\bar{p}_{1}-p_{1}$ and the sensing errors $\Delta T_{\mathrm{i}}=\bar{T}_{\mathrm{i}}-T_{\mathrm{i}}, \Delta T m=\bar{m}_{1} \bar{T}_{\mathrm{i}}-m_{1} T_{\mathrm{i}}, \Delta \dot{m}_{1}=\dot{\bar{m}}_{1}-\dot{m}_{1}$. These terms will cause a bias in the estimate of $\hat{\theta}$.

The update law is chosen to be the gradient algorithm

$$
\dot{\hat{\theta}}= \begin{cases}\Gamma \bar{\phi} \bar{\varepsilon} & \hat{\eta}_{\mathrm{v}}=\mathscr{P}_{z}(N) \hat{\theta} \in \mathscr{S}_{\eta} \quad \Gamma>0 \\ 0 & \hat{\eta}_{\mathrm{v}}=\mathscr{P}_{z}(N) \hat{\theta} \notin \mathscr{S}_{\eta}\end{cases}
$$


where $\mathscr{S}_{\eta}$ is a bounded set and defined as $\eta_{\mathrm{v}}(t) \in \mathscr{S}_{\eta}:=\left\{\eta_{\mathrm{v}} \mid 0<\eta_{\mathrm{vMIN}} \leqslant \eta_{\mathrm{v}} \leqslant \eta_{\mathrm{vMAX}}\right\} \forall t$ so that $\theta(t) \in \mathscr{S}_{\theta}(t):=\left\{\theta(t) \mid \mathscr{P}_{\rho}(N(t)) \theta(t) \in \mathscr{S}_{\eta}\right\} \subset \mathscr{S}_{\theta} \subset \mathbb{R}_{+} \forall t$.

\section{Theorem}

The adaptive air charge estimation scheme summarised as the observer (19)-(21) with the update law (36) driven by the identification error (35) and the regressor in (33) has the following properties:

(i) Assuming that there are no compressor instabilities, the state $x^{\mathrm{T}}:=\left[p_{1}, m_{1}\right]$ and the input $u^{\mathrm{T}}:=\left[T_{\mathrm{i}}, T_{1}, N\right]$ belong in bounded sets, and thus, all the errors $\tilde{\theta}, \tilde{p}_{1}, \tilde{m}_{1}, \tilde{W}_{1 \mathrm{e}}$ are bounded.

(ii) If all the sensing errors and the pressure ripples are zero, and the parameter $\theta$ is constant, then the identified parameter converges to the true constant one $(\hat{\theta} \rightarrow \theta)$ exponentially.

(iii) If we measure the compressor flow accurately in addition to conditions in (ii), then $\left(\hat{W}_{1 \mathrm{e}} \rightarrow W_{1 \mathrm{e}}\right)$ exponentially.

The proof is given in Appendix A.

A simulation of the adaptive estimation compared to the traditional air charge estimation schemes is shown in Figure 3. It performs similarly to the MAF scheme. The identification error (35) is more sensitive to the ripple $\Delta p_{1}$ and the sensing errors $\Delta \dot{m}_{1}$, etc. Since the effects caused by the sensing errors increase with the identification gain $\Gamma$, this limits the feasible values for $\Gamma$, and in turn the convergence rate of $\tilde{\theta}$. Owing to the significant difference between the temperature and pressure sensor dynamics in this scheme, the higher-order dynamics introduced by the sensors need to be accounted for if the convergence rate of $\hat{\theta}$ is to be acceptable.

This scheme is not vulnerable to possible errors in the $W_{\mathrm{cl}}$ measurement. In addition, the observer equation for $\hat{m}_{1}(20)$ is closed-loop which gives better robustness to modelling errors in the observer. These beneficial features have been traded with one more measurement, slower convergence time for $\hat{\theta}$, and most notably that $\bar{\phi}$ is not always persistently exciting.

The regressor $\bar{\phi}$ is not always persistently exciting (PE). The PE condition fails when $k_{1}^{2} \bar{T}_{\mathrm{i}} \bar{m}_{1}-\bar{p}_{1}=0$ in some time interval. If there are no sensor errors and no pressure ripples, the PE condition implies that $T_{\mathrm{i}}=\left(1 / k_{1}^{2}\right) p_{1} / m_{1}=T_{1}$ which is the case at equilibrium, where instability of $\hat{\theta}$ can occur. ${ }^{\S}$ This will make $\hat{\theta}$ drift to the boundary of the projection set $\mathscr{S}_{\theta}(t)$. Approximate tracking of the time varying $\theta(t)$ cannot be assured when the PE condition fails. One way to remedy this is to switch the adaption off when the regressor is close to zero and $k_{1}^{2} T_{\mathrm{i}} m_{1}-p_{1}$ is small.

\subsection{Unmodelled dynamics introduced by the sensors}

The sensors can be assumed to be linear first-order systems, so that $\bar{p}_{1}:=H_{p 1} p_{1}, \bar{T}_{1}:=H_{T 1} T_{1}$ and $\bar{T}_{\mathrm{i}}:=H_{T_{\mathrm{i}}} T_{\mathrm{i}}$. For example consider the $p_{1}$ measuring signal

$$
\bar{p}_{1}=\left[H_{p 1}\left(p_{1}+\Delta p_{1}\right)\right]=\frac{p_{p 1}}{s+p_{p 1}}\left(p_{1}+\Delta p_{1}\right)=\frac{1}{\tau_{p 1} s+1}\left(p_{1}+\Delta p_{1}\right)
$$

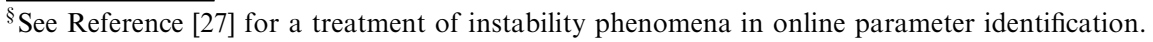


which introduces additional dynamics in the identification error $\bar{\varepsilon}$ (second term in the right-hand side of Equation (35)):

$$
\begin{gathered}
H_{\mathrm{f}}\left(\frac{\mathrm{d}}{\mathrm{d} \tau}+g_{p 1}\right) H_{p 1}\left(\bar{p}_{1}+\hat{p}_{1}\right)=\frac{s+g_{p 1}}{\left(\tau_{\omega} s+1\right)\left(\tau_{p 1} s+1\right)}\left(p_{1}+\Delta p_{1}-\left(\tau_{p 1} s+1\right) \hat{p}_{1}\right) \\
=\frac{s+g_{p 1}}{\left(\tau_{\omega} s+1\right)\left(\tau_{p 1} s+1\right)}\left(\tilde{p}_{1}+\Delta p_{1}-\tau_{p 1} s \hat{p}_{1}\right)
\end{gathered}
$$

This gives a considerable inverted initial response in $\tilde{\theta}$ from $\dot{\hat{p}}_{1}$.

The slow temperature measurement $T_{1}$ relative to the $p_{1}$ measurement creates problems for the $\bar{m}_{1}$ approximation which becomes

$$
\bar{m}_{1}=\frac{1}{k_{1}^{2}} \frac{\bar{p}_{1}}{\bar{T}_{1}}=\frac{\left[H_{p 1} p\right](t)}{\left[H_{T 1} T_{1}\right](t)}
$$

This is very much different from a slightly filtered $\left[H_{m} m_{1}\right](t)$ since the time constant of $H_{T 1}$ is much larger than the time constant of $H_{p 1}$. In addition, the sensor dynamics of $\bar{T}_{\mathrm{i}}$ further worsen the transient behaviour of $\tilde{\theta}$ and can even destroy its convergence.

The problem with the $\bar{m}_{1}$ approximation can to some extent be removed by filtering with a filter $H_{\mathrm{c}}$ so that

$$
\bar{m}_{1}:=\frac{1\left[H_{\mathrm{c}} p_{1}\right](t)}{k_{1}^{2}\left[H_{\mathrm{c}} T_{1}\right](t)}=\frac{1}{k_{1}^{2}} \frac{\left[H_{\mathrm{c}} H_{p 1}^{-1} \bar{p}_{1}\right](t)}{\left[H_{\mathrm{c}} H_{T 1}^{-1} \bar{T}_{1}\right](t)}
$$

where the bandwidth of $H_{\mathrm{c}}$ is limited by the noise level and the time constant of $T_{1}$ which is the slowest measurement. Consequently, the high-frequency information in the $p_{1}$ measurement cannot be used if the temperature measurements are significant slower, and the convergence of $\tilde{\theta}$ will be slower. Also, note that the time constant of the temperature measurements is a function of the flow $W_{\mathrm{cl}}$ which is not measured. This is a possible obstacle, but if overcome, and in addition the temperature measurements are sufficiently fast, the response of this scheme is similar to the uncompensated one in Figure 3.

\section{SIMULATION RESULTS}

To provide a bit of insight on the practicality of the above analysis we use a mean value model of a turbocharged 2.01 diesel engine documented in Reference [9] and consequently used for control development in References [28,29]. The adaptive air charge estimation is evaluated during fueling level steps (from 5 to $15 \mathrm{~kg} / \mathrm{h}$ at time $0.2 \mathrm{~s}$ and a negative step back to $5 \mathrm{~kg} / \mathrm{h}$ at $t=0.7 \mathrm{~s}$ ). Such a large increase in fuel flow is typically followed by opening the wastegate or the turbine nozzles in an engine equipped with variable geometry turbocharger (VGT). To match typical operating engine conditions we also vary the road load. All the input traces are shown in Figure 2.

The volumetric efficiency of the model is

$$
\eta_{\mathrm{v}}=\mathscr{P}_{m}^{3}(N)\left(1-0.003\left(p_{2}^{\prime}-p_{1}\right)\right) \quad \text { where } p_{2}^{\prime}(t)=p_{2}(t-\delta(t))
$$

with $\delta(t)=(60 / N(t)) \frac{1}{2}$ (one event). Also, $\mathscr{P}_{m}^{3}(N)$ is a third-order polynomial representing steady state pumping as a function of engine speed. 

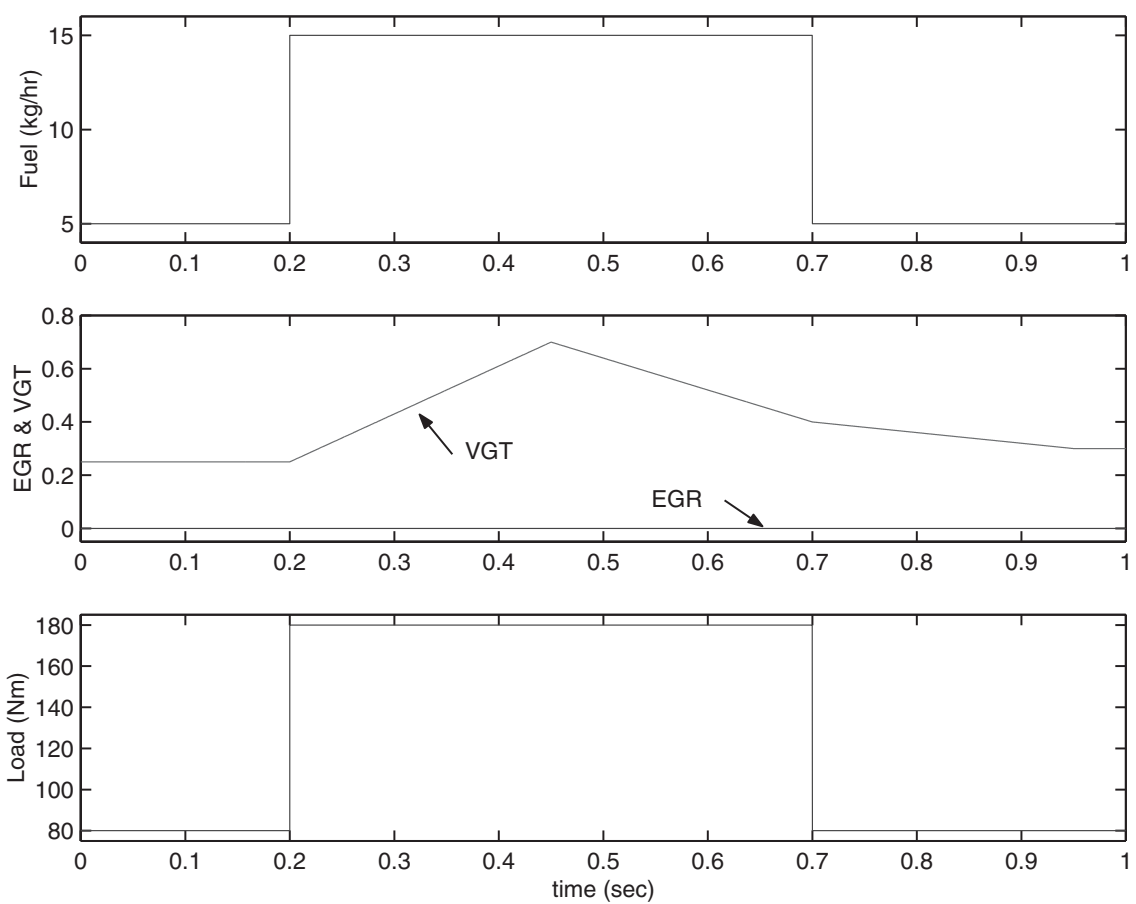

Figure 2. Inputs to the mean-value engine model.

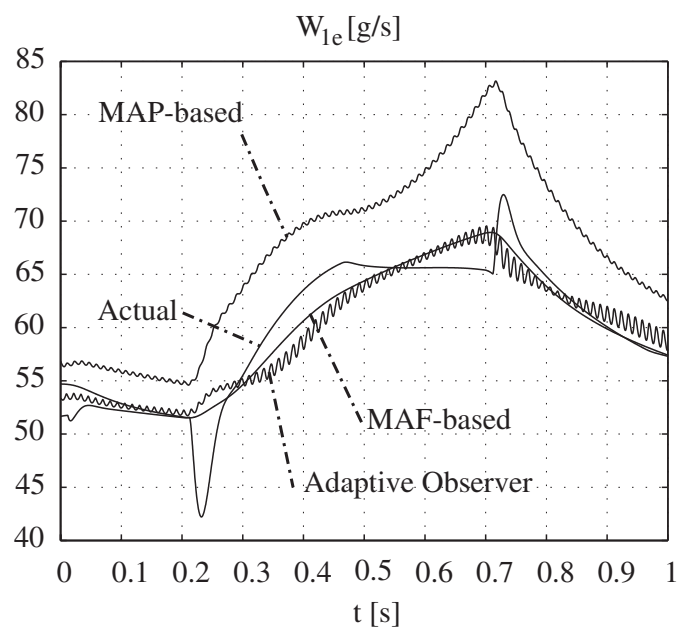

Figure 3. Engine air flow as estimated by the adaptive observer based on engine speed, intake manifold temperatures and pressure. The actual engine air flow as well as the estimated using the traditional MAP- and MAF-based estimation are shown for comparison. 
The $\eta_{\mathrm{v}}$ parameterisation for the proposed scheme is

$$
\hat{\eta}_{\mathrm{v}}=\left(\mathscr{P}_{m}^{3}(N)+0.15\right) \hat{\theta}
$$

Whereas, the pumping rate for the speed density ('MAP') scheme in Equation (8) and mass air flow ('MAF') scheme in Equation (6) is

$$
\begin{gathered}
\hat{\eta}_{\mathrm{v}}=\mathscr{P}_{\mathrm{v}}\left(N, p_{1}\right)=\left(\mathscr{P}_{m}^{3}(N)+0.15\right)\left(1-0.003\left(p_{2 \text { mean }}-p_{1}\right)\right) \\
\mathscr{P}_{w}\left(N, p_{1}, T_{1}\right)=k_{3} N \frac{p_{1}}{T_{1}} \mathscr{P}_{\mathrm{v}}\left(N, p_{1}\right)
\end{gathered}
$$

where $p_{2 \text { mean }}$ is taken to be the average value of $p_{2}$ in the simulation. In all three cases, $\hat{\eta}_{\mathrm{v}}$ has a large constant deviation of $15 \%$ from the one used in the simulation model.

The time constant of $H_{p 1}$ is $5 \mathrm{~ms}$, and the $2.5 \%$ ripple in $\bar{p}_{1}$ is represented in the model as

$$
\Delta p_{1}(t)=\left(0.127324-\left|\sin \left(\frac{2 \pi}{60} N(t)\right)\right|\right) 0.0125 p_{1}
$$

The temperature sensor time constant for the MAF schemes is $1.0 \mathrm{~s}$, whereas we assume a time constant of $0.1 \mathrm{~s}$ for the proposed (temperature-based) scheme. Although the adaptive observer has the appropriate filters for dealing with temperature sensor time constant of $0.1 \mathrm{~s}$, there are no sensor dynamics modelled when simulating the proposed scheme.

The estimation parameters for this simulation are: $\tau_{\omega}=(0.08 N)^{-1}, \Gamma=0.03, g_{p 1}=300, g_{m 1}$ $=1000, \bar{W}_{\mathrm{cl}}=0.05$. The engine air flow estimation results are shown in Figure 3. Although the proposed observer does not have the bandwidth needed to capture the large initial air excursion, it has a similar $W_{\text {le }}$ estimate with the traditional 'MAF'-based estimator that utilises a mass air flow sensor and slow temperature measurements. One should note that the comparison between the proposed and the MAF scheme is unfair due to the differences in the assumed temperature sensor dynamics. However, the comparison illustrates that robust air charge estimation is achievable even without air flow sensors if good (fast) temperature sensors are available. This robustness, reduction in engine mapping, and potential cost elimination from the elimination of the air flow sensor, comes at the expense of algorithmic challenges and elaborate sensor characterisation.

\section{CONCLUSIONS AND FUTURE WORK}

In this paper, we use a temperature measurement instead of MAF and show that in the case of zero-EGR the proposed adaptive observer is comparable in performance to the conventional 'MAF'-based air charge estimation. Advances in temperature sensor technology will greatly facilitate adaptive control and observer design in advanced technology engines because it contains additional information on the dynamics not easily recovered otherwise.

Fast temperature sensors are considered in addition to flow and pressure sensors in engines with exhaust gas recirculation. Exhaust gas recirculation gives rise to burnt gas fraction dynamics in the intake and the exhaust manifold. These dynamics are weakly observable by flow and pressure measurements of intake manifold variables [13] and require additional sensors such as temperature to enable observability. In-cylinder air estimation in engines with EGR is much harder and requires more sensors and non-trivial modifications of the proposed estimation scheme. The air charge estimation for engines with EGR will be addressed in future work. 


\section{APPENDIX A: PROOF OF THEOREM}

As we have indicated in Section 3 the implementable identification error is linear with respect to the parameter error except from some terms that depend on sensing errors and higher-order cylinder-to-cylinder dynamics. These terms can cause estimation bias or even destabilisation, we thus start the proof of (i) by identifying these terms and then quantifying their effects on the parameter error convergence.

First, let $z(t)$ in $(28)$

$$
\begin{aligned}
z & =[\bar{\phi} \theta]=-\left[H_{\mathrm{f}} G_{p N}\left(k_{1}^{2} \bar{m}_{1} \bar{T}_{\mathrm{i}}-\bar{p}_{1}\right) \theta\right] \\
& =-\left[H_{\mathrm{f}}\left(G_{p N}\left(k_{1}^{2} m_{1} T_{\mathrm{i}}-p_{1}\right) \theta-G_{p N} k_{1}^{2}\left(m_{1} T_{\mathrm{i}}-\bar{m}_{1} \bar{T}_{\mathrm{i}}\right) \theta+G_{p N}\left(p_{1}-\bar{p}_{1}\right) \theta\right)\right] \\
& =-\left[H_{\mathrm{f}} G_{p N}\left(k_{1}^{2} m_{1} T_{\mathrm{i}}-p_{1}\right) \theta\right]-\left[H_{\mathrm{f}} G_{p N}\left(k_{1}^{2} \Delta T m+\Delta p_{1}\right) \theta\right] \\
& =\left[H_{\mathrm{f}}\left(\kappa k_{1}^{2} \dot{m}_{1} T_{\mathrm{i}}-\dot{p}_{1}\right)\right]-\left[H_{\mathrm{f}} G_{p N}\left(k_{1}^{2} \Delta T m+\Delta p_{1}\right) \theta\right]
\end{aligned}
$$

using Equations (22)-(24)

Similarly, $\hat{z}(t)$ from (29) can be implemented as

$$
\begin{aligned}
\hat{z}=[\bar{\phi} \hat{\theta}]= & -\left[H_{\mathrm{f}} G_{p N}\left(k_{1}^{2} \bar{m}_{1} \bar{T}_{\mathrm{i}}-\bar{p}_{1}\right) \hat{\theta}\right](t) \\
= & -\left[H_{\mathrm{f}} \kappa k_{1}^{4} k_{3} N \mathscr{P}_{z}(N) \bar{m}_{1} \bar{T}_{\mathrm{i}} \hat{\theta}\right]+\left[H_{\mathrm{f}} \kappa k_{1}^{2} k_{3} N \mathscr{P}_{z}(N) \bar{p}_{1} \hat{\theta}\right] \\
= & {\left[H_{\mathrm{f}} \kappa k_{1}^{2} \bar{T}_{\mathrm{i}}\left(\dot{\hat{m}}_{1}-W-g_{m 1}\left(\bar{m}_{1}-\hat{m}_{1}\right)\right)\right] } \\
& -\left[H_{\mathrm{f}}\left(\dot{\hat{p}}_{1}-\kappa k_{1}^{2} \bar{T}_{\mathrm{i}} W-g_{p 1}\left(\bar{p}_{1}-\hat{p}_{1}\right)\right)\right] \\
= & {\left[H_{\mathrm{f}} \kappa k_{1}^{2} \bar{T}_{\mathrm{i}}\left(\dot{\hat{m}}_{1}-g_{m 1}\left(\bar{m}_{1}-\hat{m}_{1}\right)\right)\right]-\left[H_{\mathrm{f}}\left(\dot{\hat{p}}_{1}-g_{p 1}\left(\bar{p}_{1}-\hat{p}_{1}\right)\right)\right] }
\end{aligned}
$$

where, we used (19)-(20) to derive (A7) from (A6) above.

Manipulating (A4) and (A8) and utilising the sensing error definitions $\Delta T_{\mathrm{i}}=\bar{T}_{\mathrm{i}}-T_{\mathrm{i}}, \Delta T m=$ $\bar{m}_{1} \bar{T}_{\mathrm{i}}-m_{1} T_{\mathrm{i}}, \Delta \dot{m}_{1}=\dot{\bar{m}}_{1}-\dot{m}_{1}$ and the pressure ripples $\Delta p_{1}=\bar{p}_{1}-p_{1}$ we derive the identification error as

$$
\begin{aligned}
\varepsilon= & z(t)-\hat{z} \\
= & \bar{\varepsilon}(t)=\kappa k_{1}^{2}\left[H_{\mathrm{f}} \bar{T}_{\mathrm{i}}\left(\frac{\mathrm{d}}{\mathrm{d} \tau}+g_{m 1}\right)\left(\bar{m}_{1}-\hat{m}_{1}\right)\right]-\left[H_{\mathrm{f}}\left(\frac{\mathrm{d}}{\mathrm{d} \tau}+g_{p 1}\right)\left(\bar{p}_{1}-\hat{p}_{1}\right)\right] \\
& -\kappa k_{1}^{2}\left[H_{\mathrm{f}}\left(\bar{T}_{\mathrm{i}} \Delta \dot{m}_{1}+\Delta T_{\mathrm{i}} \dot{m}_{1}\right)\right]+\left[H_{\mathrm{f}} \frac{\mathrm{d}}{\mathrm{d} \tau} \Delta p_{1}\right] \\
& -\left[H_{\mathrm{f}} G_{p N}\left(k_{1}^{2} \Delta T m+\Delta p_{1}\right) \theta\right]
\end{aligned}
$$


If we neglect the terms in the third line of (A9), this results in the implementable identification error in (35) or more specifically:

$$
\begin{aligned}
\bar{\varepsilon}= & \varepsilon-\kappa k_{1}^{2}\left[H_{\mathrm{f}}\left(\bar{T}_{\mathrm{i}} \Delta \dot{m}_{1}+\Delta T_{\mathrm{i}} \dot{m}_{1}\right)\right]+\left[H_{\mathrm{df}} \Delta p_{1}\right]-\left[H_{\mathrm{f}} G_{p N}\left(k_{1}^{2} \Delta T m+\Delta p_{1}\right) \theta\right] \\
= & \bar{\phi} \tilde{\theta}-\left[H_{\mathrm{s}} \bar{\phi}(\tau) \dot{\tilde{\theta}}(\tau)\right]-\kappa k_{1}^{2}\left[H_{\mathrm{f}}\left(\bar{T}_{\mathrm{i}} \Delta \dot{m}_{1}+\Delta T_{\mathrm{i}} \dot{m}_{1}\right)\right]+\left[H_{\mathrm{df}} \Delta p_{1}\right] \\
& -\left[H_{\mathrm{f}} G_{p N}\left(k_{1}^{2} \Delta T m+\Delta p_{1}\right) \theta\right]
\end{aligned}
$$

where we used (31) to explicitly include the regressor $\bar{\phi}$ and the parameter error $\tilde{\theta}$ in the implementable identification error $\bar{\varepsilon}$. We also employed the simplified notation of the filter $\left[H_{\mathrm{df}} u\right]=\left[H_{\mathrm{f}}(\mathrm{d} / \mathrm{d} \tau) u(\tau)\right]:$

$$
\left[H_{\mathrm{df}} u\right](t)=x_{\mathrm{df}}+k_{\omega} N u \quad \text { with } \dot{x}_{\mathrm{df}}=-k_{\omega} N x_{\mathrm{df}}-k_{\omega}^{2} N^{2} u
$$

Using (36) we define the rate of change of the parameter error:

$$
\begin{aligned}
\dot{\tilde{\theta}}= & \dot{\theta}-\Gamma \bar{\phi}\left(\bar{\phi} \tilde{\theta}-\left[H_{\mathrm{s}} \bar{\phi}(\tau) \dot{\tilde{\theta}}(\tau)\right]-\left[H_{\mathrm{df}} \Delta p_{1}\right]+\left[H _ { \mathrm { f } } \left(\kappa k_{1}^{2}\left(\bar{T}_{\mathrm{i}} \Delta \dot{m}_{1}+\Delta T_{\mathrm{i}} \dot{m}_{1}\right)\right.\right.\right. \\
& \left.\left.\left.+G_{p N}\left(k_{1}^{2} \Delta T m+\Delta p_{1}\right) \theta\right)\right]\right)
\end{aligned}
$$

By expanding the filter notation (25), (34) and (A11) and combining them into the dynamical state $x_{\mathrm{a}}$ we can derive the following second-order system:

$$
\begin{aligned}
{\left[\begin{array}{c}
\dot{x}_{\mathrm{a}} \\
\dot{\tilde{\theta}}
\end{array}\right]=} & {\left[\begin{array}{cc}
-k_{\omega} N+\Gamma \bar{\phi}^{2} & \Gamma \bar{\phi}^{3} \\
-\Gamma \bar{\phi} & -\Gamma \bar{\phi}^{2}
\end{array}\right]\left[\begin{array}{c}
x_{\mathrm{a}} \\
\tilde{\theta}
\end{array}\right] } \\
+ & {\left[\begin{array}{ccc}
k_{\omega} N & k_{\omega} N\left(\Gamma \bar{\phi}^{2}+k_{\omega} N\right) & -\bar{\phi} \\
0 & -k_{\omega} N \Gamma \bar{\phi} & 1
\end{array}\right]\left[\begin{array}{c}
f_{\mathrm{se}}\left(\Delta T_{\mathrm{i}}, \Delta \dot{m}_{1}, \Delta T m\right) \\
\Delta p_{1} \\
\dot{\theta}
\end{array}\right] }
\end{aligned}
$$

where the term that corresponds to all the sensor errors is $f_{\mathrm{se}}\left(\Delta T_{\mathrm{i}}, \Delta \dot{m}_{1}, \Delta T m\right)=\kappa k_{1}^{2} \times$ $\left(\bar{T}_{\mathrm{i}} \Delta \dot{m}_{1}+\Delta T_{\mathrm{i}} \dot{m}_{1}\right)+k_{1}^{2} G_{p N} \Delta \operatorname{Tm} \theta$. The linear time-varying system in (A13) that can be expressed as $\dot{x}=A(t) x+B(t) u$ is exponentially stable since the eigenvalues of $A(t)+A^{\mathrm{T}}(t)$ can satisfy

$$
\int_{t_{0}}^{t} \lambda_{\max \left(A+A^{\mathrm{T}}\right)}(\tau) \mathrm{d} \tau \leqslant-\lambda\left(t-t_{0}\right)+\mu \quad \forall t>t_{0}
$$

for some positive constant $\lambda$ and $\mu$. If we assume bounded sensing errors one also can show that $B(t) u(t)$ is bounded. We thus conclude that the parameter error $\tilde{\theta}$ is bounded.

Owing to the estimation error injection in Equations (19) and (20) the dynamical systems for $\hat{p}_{1}$ and $\hat{m}_{1}$ are stable and their input is bounded, thus, the air flow error $\tilde{W}_{1 \mathrm{e}}$ is bounded.

\section{A.1. Zero sensing errors}

If the pressure ripples $\Delta p_{1}=\bar{p}_{1}-p_{1}$ and the sensing errors $\Delta T_{\mathrm{i}}=\bar{T}_{\mathrm{i}}-T_{\mathrm{i}}, \Delta T m=\bar{m}_{1} \bar{T}_{\mathrm{i}}-$ $m_{1} T_{\mathrm{i}}, \Delta \dot{m}_{1}=\dot{\bar{m}}_{1}-\dot{m}_{1}$ are all zero, then the $\bar{\varepsilon}=\varepsilon$ and thus the only bias for the parameter error will depend on the swapping error in Equation (32):

$$
\dot{\tilde{\theta}}=\dot{\theta}-\Gamma \bar{\phi}\left(\bar{\phi} \tilde{\theta}-\left[H_{\mathrm{s}} \bar{\phi}(\tau) \dot{\tilde{\theta}}(\tau)\right]\right)
$$


By expanding the filter $H_{\mathrm{S}}$ to its state space representation we obtain

$$
\begin{gathered}
\dot{\tilde{\theta}}=\dot{\theta}-\Gamma \bar{\phi}^{2} \tilde{\theta}+\Gamma \bar{\phi} x_{\mathrm{s}} \\
\dot{x}_{\mathrm{s}}=-k_{\omega} N x_{\mathrm{s}}+\bar{\phi} \dot{\tilde{\theta}}=-k_{\omega} N x_{\mathrm{s}}+\bar{\phi}\left[\dot{\theta}-\Gamma \bar{\phi}^{2} \tilde{\theta}+\Gamma \bar{\phi} x_{\mathrm{s}}\right]
\end{gathered}
$$

and its state space form

$$
\left[\begin{array}{c}
\dot{x}_{\mathrm{s}} \\
\dot{\tilde{\theta}}
\end{array}\right]=\left[\begin{array}{cc}
-k_{\omega} N+\Gamma \bar{\phi}^{2} & -\Gamma \bar{\phi}^{3} \\
\Gamma \bar{\phi} & -\Gamma \bar{\phi}^{2}
\end{array}\right]\left[\begin{array}{c}
x_{\mathrm{s}} \\
\tilde{\theta}
\end{array}\right]+\left[\begin{array}{c}
-\bar{\phi} \\
1
\end{array}\right] \dot{\theta}
$$

which can be expressed as $\dot{x}=A_{n \mathrm{~s}} x+B \dot{\theta}$ and has stable dynamics because the eigenvalues satisfy (A14) since $\lambda_{\left(A_{n \mathrm{~s}}+A_{n \mathrm{~s}}^{\mathrm{T}}\right)}=\lambda_{\left(A+A^{\mathrm{T}}\right)}$. As a result, $\tilde{\theta}$ is bounded if $\dot{\theta}$ is bounded. Moreover, $\tilde{\theta} \rightarrow 0$ if $\dot{\theta}=0$ (time invariant volumetric efficiency and correctly parameterised).

\section{A.2. Measured compressor air flow}

If there are no pressure ripples and no sensor errors it is easy to show that

$$
\begin{gathered}
\dot{\tilde{p}}_{1}=-g_{p 1} \tilde{p}_{1}-\kappa k_{1}^{2} k_{3} N \mathscr{P}_{z}(N) p_{1} \tilde{\theta}+\kappa k_{1}^{2} T_{\mathrm{i}}\left(W_{\mathrm{cl}}-W\right) \\
\dot{\tilde{m}}_{1}=-g_{p 1} \tilde{m}_{1}-k_{1}^{2} k_{3} N \mathscr{P}_{z}(N) m_{1} \tilde{\theta}+\left(W_{\mathrm{c} 1}-W\right) \\
\tilde{W}_{1 \mathrm{e}}=k_{1} k_{3} N \mathscr{P}_{z}(N)\left(\theta \tilde{m}_{1}+\tilde{\theta} \hat{m}_{1}\right)
\end{gathered}
$$

If the true volumetric efficiency is given by (9), i.e. $\mathscr{P}_{\rho}=\mathscr{P}_{z}$ with constant parameter $\theta$, and the compressor flow is measured $\left(W=W_{\mathrm{c} 1}\right)$, it is easy to show that $\tilde{p}_{1} \rightarrow 0, \tilde{m}_{1} \rightarrow 0$ and $\tilde{W}_{1 \mathrm{e}} \rightarrow 0$.

\section{APPENDIX B: NOMENCLATURE}

$\begin{array}{ll}k_{\omega} & \text { filter coefficient } \\ m_{1} & \text { mass of gas in the intake manifold }(\mathrm{kg}) \\ m_{2} & \text { mass of gas in the exhaust manifold }(\mathrm{kg}) \\ N & \text { engine speed }(\mathrm{rpm}) \\ p_{1} & \text { pressure in the intake manifold }(\mathrm{kPa}) \\ p_{2} & \text { pressure in the exhaust manifold }(\mathrm{kPa}) \\ p_{\mathrm{a}} & \text { ambient pressure }(\mathrm{kPa}) \\ R_{1} & \text { gas constant for the intake manifold }(\mathrm{kJ} /(\mathrm{kg} \mathrm{K}) \\ r & \text { engine compression ratio }(\text { dimensionless }) \\ T_{1} & \text { temperature of gas in the intake manifold }(\mathrm{K}) \\ T_{2} & \text { temperature of gas in the exhaust manifold }(\mathrm{K}) \\ T_{\mathrm{a}} & \text { ambient temperature }(\mathrm{K}) \\ T_{\mathrm{c}} & \text { temperature of the air leaving the compressor }(\mathrm{K}) \\ T_{\mathrm{i}} & \text { temperature of the gas leaving the intercooler }(\mathrm{K}) \\ V_{1} & \text { volume of the intake manifold }\left(\mathrm{m}^{3}\right) \\ V_{\mathrm{d}} & \text { total engine displacement volume }\left(\mathrm{m}^{3}\right) \\ W_{\mathrm{le}} & \text { mass flow into the engine }(\mathrm{kg} / \mathrm{s}) \\ W_{\mathrm{cl}} & \text { mass flow from compressor to intake manifold }(\mathrm{kg} / \mathrm{s})\end{array}$




$\begin{array}{ll}W_{\mathrm{t}} & \text { throttle mass flow }(\mathrm{kg} / \mathrm{s}) \\ W_{\mathrm{f}} & \text { engine fueling level }(\mathrm{kg} / \mathrm{h}) \\ \Gamma & \text { identification gain for the gradient algorithm see Equation (36) (dimensionless) } \\ \varepsilon \text { and } \bar{\varepsilon} & \text { identification error and implementable identification error } \\ \epsilon_{\mathrm{s}} & \text { swapping error see Equation (32) } \\ \eta_{\mathrm{v}} & \text { volumetric efficiency of the engine (dimensionless) } \\ \phi \text { and } \bar{\phi} & \text { regressor signal and implementable regressor signal }\end{array}$

\section{ACKNOWLEDGEMENTS}

Support for Stefanopoulou and Storset is provided by the National Science Foundation under contract NSF-ECS-0049025 and Ford Motor Company through a 2001 University Research Project; Smith is supported by the National Science Foundation under contract NSF-ECS-9978562.

\section{REFERENCES}

1. Shulman MA, Hamburg DR, Throop MJ. Comparison of measured and predicted three-way catalyst conversion effciencies under dynamic air-fuel ratio conditions. SAE Technical Paper 820276, 1982.

2. Turin RC, Geering HP. On-line identification of air-fuel ratio dynamics in a sequentially injected si engine. $S A E$ Technical Paper 930857, 1993.

3. Grizzle JW, Dobbins KL, Cook JA. Individual cylinder air-fuel ratio control with a single EGO sensor. IEEE Transactions on Vehicular Technology 1991; 40(1):280-286.

4. Mooncheol W, Choi SB, Hedrick JK. Air-to-fuel ratio control of spark ignition engines using gaussian network sliding control. IEEE Transactions on Control Systems Technology 1998; 6(5):678-687.

5. Powell JD, Fekete NP, Chang C-F. Observer-based air fuel ratio control. IEEE Control Systems Magazine 1998; 18(5):72-83.

6. Fukutani I, Watanabe E, Komotori K, Miyake K. Throttling of 2-stroke cycle diesel engines at part-load and idling. SAE Technical Paper 730187, 1973.

7. Guzzella L, Amstutz A. Control of diesel engines. IEEE Control Systems Magazine 1998; 18(2):53-71.

8. Hendricks E. Mean value modelling of large turbocharged two-stroke diesel engine. SAE Technical Paper 890564, 1989.

9. Kolmanovsky IV, Moral PE, van Nieuwstadt M, Stefanopoulou AG. Issues in modelling and control of intake flow in variable geometry turbocharged engines. Proceedings of the 18th IFIP Conference on System Modelling and Optimisation, Detroit, MI, vol. 1, July 1998, 436-445.

10. Taylor CF. The Internal-Combustion Engine in Theory and Practice. M.I.T. Press: Cambridge, MA, 1985.

11. Yuen WW, Servati H. A mathematical engine model including the effect of engine emissions. SAE Technical Paper 840036, 1984.

12. Kolmanovsky IV, Jankovic M, van Nieuwstadt M, Moraal PE. Method of estimating mass air flow in turbocharged engines having exhaust gas recirculation. U.S. Patent 6,035,639, 2000.

13. Diop S, Moral PE, Kolmanovsky IV, van Nieuwstadt M. Intake oxygen concentration estimation for DI diesel engines. Proceedings of the 1999 IEEE International Conference on Control Applications, Kohala Coast, Hawaii, vol. 1,$1999 ; 852-857$.

14. Amstutz A, Del Re LR. EGO sensor based robust output control of EGR in diesel engines. IEEE Transactions on Control System Technology 1995; 3(1):39-48.

15. Kolmanovsky IV, Sun J, Druzhinina M. Charge control for direct injection spark ignition diesel engines with EGR. Proceedings of the 2000 American Control Conference, Chicago, IL, vol. 1, 2000; 34-38.

16. Stotsky A, Kolmanovsky IV. Simple unknown input estimation techniques for automotive applications. Proceedings of the 2001 American Control Conference, Arlington, VA, vol. 5, 2001; 3312-3317.

17. Andersson P, Eriksson L. Air-to-cylinder observer on a turbocharged si-engine with wastegate. SAE Technical Paper 2001-01-0262, 2001.

18. Storset OF. Air charge estimation for turbocharged diesel engines. Master's Thesis, University of California Santa Barbara, 2000. 
19. Storset OF, Stefanopoulou AG, Smith R. Air charge estimation for turbocharged diesel engines. Proceedings of the 2000 American Control Conference, Chicago, IL, vol. 1, 2000; 28-30.

20. Heywood JB. Internal Combustion Engine Fundamentals. McGraw-Hill: New York, 1988.

21. Hendricks E, Sorenson SC. Mean value modeling of spark ignition engines. SAE Technical Paper 900616, 1990.

22. Grizzle JW, Cook JA, Milam WP. Improved cylinder air charge estimation for transient air-to-fuel ratio control. Proceedings of the 1994 American Control Conference, Baltimore, OH, vol. 2, 1994; 1568-1573.

23. MEDTHERM Corporation. Coaxial thermocouples with 1 microsecond response. Bulletin 500, March 2002.

24. Muller M, Hendricks E, Sorenson SC. Mean value modelling of turbocharged spark ignition engines. SAE Technical Paper Series 980784, 1998.

25. Hermann R, Krener AJ. Nonlinear controllability and observability. IEEE Transactions on Automatic Control 1977; 22:728-740.

26. Morse AS. Global stability of parameter adaptive control systems. IEEE Transactions on Automatic Control 1980; 25:433-439.

27. Ioannou PA, Sun J. Robust Adaptive Control. Prentice-Hall: New Jersey, 1996.

28. Stefanopoulou AG, Kolmanovsky IV, Freudenberg JS. Control of a variable geometry turbocharged diesel engine for reduced emissions. IEEE Transactions on Control Systems Technology 2000; 8(4):733-745.

29. van Nieuwstadt M, Kolmanovsky IV, Moraal PE, Stefanopoulou AG, Jankovic M. EGR-VGT Control schemes: Experimental comparison for a high-speed diesel engine. IEEE Control System Magazine 2000; 20(3):63-79. 